\title{
Soft silicone elastomers with no chemical cross-linking and unprecedented softness and stability
}

Hu, Pengpeng; Huang, Qian; Jeppe Madsen, Peter; Skov, Anne Ladegaard

Published in:

Electroactive Polymer Actuators and Devices (EAPAD) XXII

Link to article, DOI:

$10.1117 / 12.2557003$

Publication date:

2020

Document Version

Publisher's PDF, also known as Version of record

Link back to DTU Orbit

Citation (APA):

Hu, P., Huang, Q., Jeppe Madsen, P., \& Skov, A. L. (2020). Soft silicone elastomers with no chemical crosslinking and unprecedented softness and stability. In Y. Bar-Cohen, I. A. Anderson, \& H. R. Shea (Eds.), Electroactive Polymer Actuators and Devices (EAPAD) XXII [1137517] SPIE - International Society for Optical Engineering. Proceedings of SPIE - The International Society for Optical Engineering Vol. 11375 https://doi.org/10.1117/12.2557003

\section{General rights}

Copyright and moral rights for the publications made accessible in the public portal are retained by the authors and/or other copyright owners and it is a condition of accessing publications that users recognise and abide by the legal requirements associated with these rights.

- Users may download and print one copy of any publication from the public portal for the purpose of private study or research.

- You may not further distribute the material or use it for any profit-making activity or commercial gain

- You may freely distribute the URL identifying the publication in the public portal 


\section{Soft silicone elastomers with no chemical cross-linking and unprecedented softness and stability}

Hu, Pengpeng, Huang, Qian, Madsen, Jeppe, Ladegaard Skov, Anne

Pengpeng Hu, Qian Huang, Jeppe Madsen, Anne Ladegaard Skov, "Soft silicone elastomers with no chemical cross-linking and unprecedented softness and stability," Proc. SPIE 11375, Electroactive Polymer Actuators and Devices (EAPAD) XXII, 1137517 (24 April 2020); doi: 10.1117/12.2557003

Event: SPIE Smart Structures + Nondestructive Evaluation, 2020, Online Only, California, United States 


\title{
Soft silicone elastomers with no chemical cross-linking and unprecedented softness and stability
}

\author{
Pengpeng $\mathrm{Hu}^{\mathrm{a}}$, Qian Huanga ${ }^{\mathrm{a}}$, Jeppe Madsen ${ }^{\mathrm{a}}$, Anne Ladegaard Skov*a \\ ${ }^{a}$ Department of Chemical and Biochemical Engineering, Technical University of Denmark, 2800 \\ Lyngby, Denmark \\ *E-mail: al@kt.dtu.dk
}

\begin{abstract}
For silicone elastomers used as actuators, softness is key for enabling actuation at low voltages. Recently, an extremely soft (Young's modulus < $50 \mathrm{kPa}$ ) silicone elastomer without cross-links has been reported by Goff et al. Besides its extreme softness, the elastomer was reported to almost completely recover $(82 \%)$ from a 10-cycle elongation of more than 5000\%. This observation challenges conventional elasticity theory of cross-linked elastomers because a network without covalent crosslinks should not be able to strain-recover to such extent. In this work, the elastomer is hypothesized to be formed from concatenated rings through heterodifunctional uni-molecular ring closure. It is found that the elastic properties of this uncross-linked elastomer can be described by the dynamics of concatenated rings, which act as pseudo-crosslinks and pseudo-entanglements. Isolated rings and dangling rings function as external solvents and internal solvents respectively, thereby contributing to the unprecedented softness. The ability to precisely control the ratio between concatenated and dangling rings is expected to lead to even softer dielectric elastomers paving the way forward for ultra-soft robotics without significant mechanical losses.
\end{abstract}

Keywords: silicone elastomer, softness, no crosslinking, concatenated ring

\section{INTRODUCTION}

Silicone elastomers are regarded as state-of-the-art elastomers for soft robotics due to their inherent flexibility, robustness, biocompatibility and high electrical insulation[1], [2]. When used as actuators, softer silicone elastomers enable deformations with less electrical energy. Thus, tailoring them towards the softness of human hard tissues $(Y>100$ $\mathrm{kPa})$ and ultimately soft tissues $(Y<1 \mathrm{kPa})$ is key for enabling actuation at low voltages and thereby providing a safer use of soft robotic actuators and enabling artificial muscle applications[3]-[5]. Typically, silicone elastomers are produced by covalent crosslinking of entangled linear polydimethylsiloxane (PDMS) polymers[6], [7]. Their lower achievable Young's moduli $(Y)$ are limited by the so-called entanglement threshold (around 0.6 MPa)[8]. Although there are examples of covalently crosslinked systems with lower Young's moduli, the resulting elastomers usually become of a very dissipative nature (see below). Therefore, producing soft silicone elastomers with negligible viscous losses is becoming a focus area in dielectric actuator research.

Soft silicone elastomers have been realized through a number of approaches, including addition of solvents[9]-[13], sparse crosslinking[14]-[16], interpenetrating networks[17], and crosslinking bottle-brush PDMS[18], [19]. Young' s moduli of silicone elastomers were lowered by adding solvents before crosslinking due to the entanglement dilution effect[20], [21]. Elastomers with tailored softness can be easily realized by this method but free species (solvents) may migrate and cause changes in the elastomer structures over time[9]. The sparse crosslinking was introduced by decreasing the ratio of cross-linker to precursor polymer[14]. This led to soft elastomers due to a large fraction of inactive junctions and dangling chains in the networks. Usually, these elastomers have a large fraction of unreacted and disconnected species in the networks, which may render them unstable over time. Excellent softness was achieved by crosslinking bottle-brush PDMS[19]. The resulting elastomers remain stable but the synthesis of bottle-brush PDMS is relatively complicated, compared with the two other approaches.

Recently, an extremely soft silicone elastomer $(Y<50 \mathrm{kPa})$ without covalent cross-links has been reported[22]. The elastomer is much softer than conventional silicone elastomers and as soft as human muscles $(Y=10-50 \mathrm{kPa}[23])$. Besides the extreme softness, it shows an excellent strain recovery from extreme elongation $(>5000 \%)$, namely $82 \%$ after 10

Electroactive Polymer Actuators and Devices (EAPAD) XXII, edited by Yoseph Bar-Cohen,

lain A. Anderson, Herbert R. Shea, Proc. of SPIE Vol. 11375, 1137517 · @ 2020

SPIE · CCC code: $0277-786 \mathrm{X} / 20 / \$ 21 \cdot$ doi: $10.1117 / 12.2557003$ 
cycles[22]. Unlike with conventional cross-linked elastomers prepared from telechelic vinyl PDMS, hydride crosslinkers and catalysts, this elastomer was produced solely using near-monodisperse $\alpha$-monovinyl- $\omega$-monohydride terminated PDMS and a platinum catalyst, without using a multifunctional cross-linker. This observation challenges conventional elasticity theory of cross-linked elastomers (illustrated by Figure 1b), because a network without covalent crosslinks should not be able to recover a strain to such extent[21].

Concatenated rings are compounds consisting of two or more mechanically interlocked cyclic polymers (rings) that cannot be destroyed without breaking at least one of the covalent bonds of the rings[24]. Since the rings can be formed from $\alpha$-monovinyl- $\omega$-monohydride terminated PDMS through intramolecular reaction, it is hypothesized that the concatenated rings are the dominant component in the highly deformable novel silicone elastomer, as shown in Figure 1a. In order to substantiate the hypothesis, tensile testing, long-term swelling experiments, rheological analysis and modeling of stress-strain were employed. Tensile tests were conducted to demonstrate the softness and the stretch-ability of the elastomer. Long-term swelling experiments and rheological measurements were performed to study the dynamic properties of the elastomer. Furthermore, the stress-strain relationship was compared to predictions based on the affine network model[25] and the slip-link network model[26].

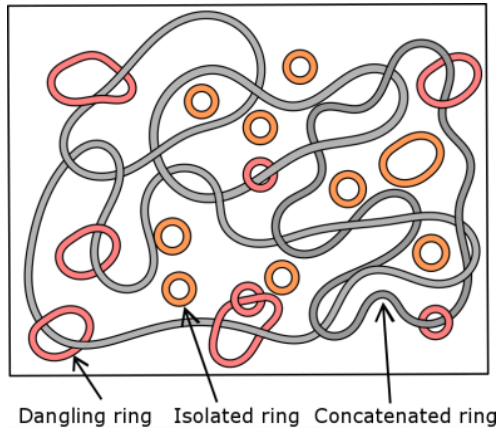

(a)

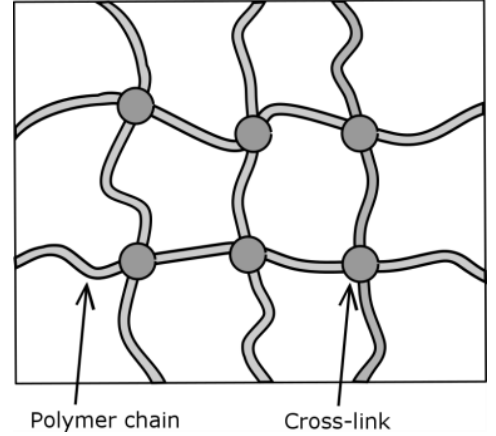

(b)

Figure 1. (a) Concatenated ring network, (b) Conventional cross-linked network.

\section{EXPERIMENTS}

\subsection{Materials}

The information of chemicals used in this study are shown in Table 1.

Table 1. Information of chemicals used in this study

\begin{tabular}{|c|c|c|c|c|c|}
\hline Chemical & Abbreviation & $\mathbf{M}_{\mathbf{n}}$ & $\boldsymbol{Ð}_{\mathrm{M}}$ & $\begin{array}{l}\text { Chemical } \\
\text { structure }\end{array}$ & Company \\
\hline $\begin{array}{l}\alpha \text {-monovinyl- } \omega \text {-monohydride } \\
\text { terminated PDMS }\end{array}$ & DMS-HV15 & $4 \mathrm{kDa}$ & 1.1 & 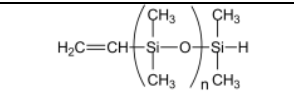 & Gelest Inc. \\
\hline Vinyl terminated PDMS & DMS-V25 & $15 \mathrm{kDa}$ & 1.5 & & Gelest Inc. \\
\hline $\begin{array}{l}\text { Dimethylsiloxane copolymer, } 23 \mathrm{wt} \% \\
\text { methylhydrosiloxane }\end{array}$ & HMS-301 & $1.9 \mathrm{kDa}$ & - & 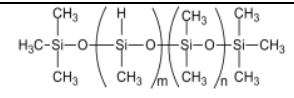 & Gelest Inc \\
\hline $\begin{array}{l}\text { Platinum-divinyl tetramethyldisiloxane } \\
\text { complex, } 3.0 \mathrm{wt} \% \mathrm{Pt}\end{array}$ & SIP 6830.3 & - & - & - & Gelest Inc. \\
\hline $\begin{array}{l}\text { Platinum cyclo-vinylmethyl siloxane } \\
\text { complex, } 1.0 \mathrm{wt} \% \mathrm{Pt}\end{array}$ & Catalyst 511 & - & - & - & $\begin{array}{l}\text { Hanse } \\
\text { Chemie }\end{array}$ \\
\hline
\end{tabular}




\subsection{Sample preparation}

The elastomer was prepared by following the procedure reported by Goff et al[22]. The precursor polymer DMS-HV15 $\left(10 \mathrm{~g}, \sim 2.5 \times 10^{-3} \mathrm{~mol}\right)$ was homogenously mixed with platinum-complex SIP $6830.3\left(10 \mathrm{mg}, 1.5 \times 10^{-6} \mathrm{~mol}\right)$ using a speed mixer (DAC 150.1 FVZ-K, Synergy Devices Ltd, UK) for $1 \mathrm{~min}$ at $3500 \mathrm{rpm}$. The mixture was then poured into a mold and cured at $80{ }^{\circ} \mathrm{C}$ for $10 \mathrm{~h}$. The obtained elastomer was abbreviated as Elastomer-HV15.

A covalently cross-linked elastomer was fabricated as a reference elastomer. Before the curing reaction, two mixtures, part A and part B, were prepared: Part A was prepared by mixing polymer DMS-V25 (5 g, 3.3 $\left.\times 10^{-4} \mathrm{~mol}\right)$ with crosslinker HMS-301 $\left(0.69 \mathrm{~g}, \sim 3.6 \times 10^{-4} \mathrm{~mol}\right)$ using a speed mixer at $3000 \mathrm{rpm}$ for $2 \mathrm{~min}$. Part B was prepared by mixing DMS-V25 (5 g, $\left.\sim 3.3 \times 10^{-4} \mathrm{~mol}\right)$ with platinum complex catalyst $511\left(20 \mathrm{mg}, 1.0 \times 10^{-7} \mathrm{~mol}\right)$ using a speed mixer. Part A and part B were mixed homogenously using speed mixer at $3000 \mathrm{rpm}$ for $30 \mathrm{~s}$. The final homogeneous mixture, with a stoichiometric imbalance (the ratio between the number of hydride groups to the number of vinyl groups) equal to $\mathrm{r}=2$, was poured into a mold and placed in an oven at $120^{\circ} \mathrm{C}$ for $10 \mathrm{~h}$. The obtained elastomer was abbreviated as ElastomerV25.

\subsection{Tensile test}

Stress-strain curves of the elastomers were determined by employing a universal testing system (Instron 3340 series, Instron, US). Specimens were cut into a dumbbell shape according to ASTM D-638 Type V (a width of $3.18 \mathrm{~mm}$, a length of $9.53 \mathrm{~mm}$ and a thickness of $1 \mathrm{~mm}$ ), then they were stretched at a crosshead speed of $500 \mathrm{~mm} / \mathrm{min}$. Young's modulus was determined by linear fitting of the experimental stress-strain data at strains of $0-10 \%$. Each sample was subjected to 3 measurements, which were then averaged.

\subsection{Swelling analysis}

Around $0.1 \mathrm{~g}$ specimen (weight $=W_{\mathrm{i}}$, with 4 significant digits) was immersed in approximately $20 \mathrm{~mL}$ chloroform at room temperature. The chloroform was replaced after the first day and decanted off after the second day. Then, the swollen specimen (weight $=W_{\mathrm{s}}$ ) was washed with fresh chloroform three times. Afterwards, the specimen was dried (weight $\left.=W_{\mathrm{d}}\right)$ by evaporating the chloroform for 1 day at a room temperature under ambient pressure. In order to extract the sol fraction completely, the dried specimen was once again swollen in around $20 \mathrm{~mL}$ chloroform for an additional 4 days $(6$ days in total). The swollen specimen was then washed with fresh chloroform, and dried under the same conditions. The dried sample was subjected to the same treatments for swelling of another 6 days (12 days in total), and 6 days more afterwards (18 days in total). In each round, $W_{\mathrm{i}}, W_{\mathrm{s}}$ and $W_{\mathrm{d}}$ were recorded. The extracted fraction (wt $\left.\%\right)$ was determined by $\left(W_{\mathrm{i}}-W_{\mathrm{d}}\right) / W_{\mathrm{i}} \times 100 \%$ and the swelling ratio was determined by $\left(W_{\mathrm{s}}-W_{\mathrm{d}}\right) / W_{\mathrm{d}}$. Each sample was analyzed in triplicate.

\subsection{Rheological analysis}

The linear viscoelasticity of the elastomer was analyzed using a strain-controlled rheometer (ARES G2, TA Instruments, US) with a small amplitude oscillatory shear. The cylinder-shape sample (a thickness of $\sim 1 \mathrm{~mm}$ and a diameter of $8 \mathrm{~mm}$ ) was measured under conditions of a fixed shear strain amplitude of $2 \%$ and a frequency range of $2 \cdot 10^{-4}-10^{2} \mathrm{~Hz}$. The measurements were performed at $25^{\circ} \mathrm{C}, 100^{\circ} \mathrm{C}$ and $200^{\circ}$ under an $\mathrm{N}_{2}$ atmosphere. The time-temperature superposition was used to generate a master curve at a reference temperature of $25^{\circ} \mathrm{C}$.

\section{RESULTS AND DISCUSSION}

\subsection{Stress-strain behaviors}

Goff et al[22] reported that a silica-filled elastomer prepared from near-monodisperse $\alpha$-monovinyl- $\omega$-monohydride terminated PDMS exhibited a maximum strain exceeding 5000\% and a tensile strength higher than $10 \mathrm{MPa}$. The elastomer in this study was prepared without silica fillers in order to achieve as low an elastic modulus as possible. Figure 2 shows that the reference elastomer Elastomer-V25, a typical non-filled silicone elastomer, can be maximally stretched to $130 \%$ with a Young's modulus of $0.84 \mathrm{MPa}$. This value is consistent with reported values of Young's moduli for commercial silicone elastomers, which are in the range of 0.5-2.0MPa[27], [28]. As a comparison, ElastomerHV15 exhibits a much lower Young's modulus of $0.049 \mathrm{MPa}$, and a maximum strain of $1500 \%$. These values are comparable to the mechanical properties of the unfilled elastomers reported by Goff et al[22]. 


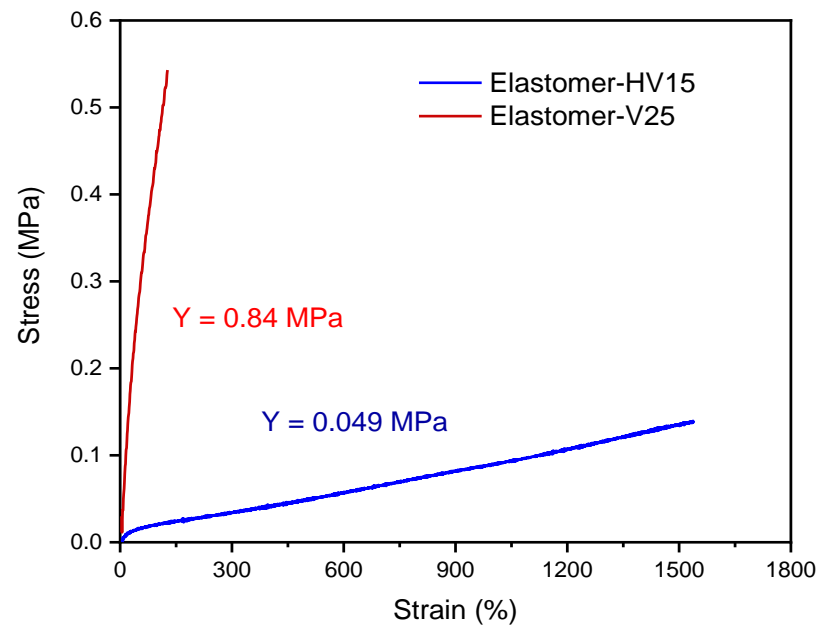

Figure 2. Stress-strain curves of the elastomer (Elastomer-HV15) and the conventional elastomer (Elastomer-V25). The original samples were cut into a dumbbell shape according to ASTM D-638 Type V (width $\times$ length $\times$ thickness $=3.18 \mathrm{~mm} \times 9.53 \mathrm{~mm} \times 1$ $\mathrm{mm}$ ). The tests were conducted at room temperature and the stretching rate was $500 \mathrm{~mm} / \mathrm{min}$.

\subsection{Long-term swelling experiment}

The long-term dynamic swelling properties of the elastomer in chloroform are shown in Figure 3. The extracted fraction gradually reaches a plateau value of $30 \%$ after 2 days. The swelling ratio behaves similar to the extracted fraction, showing a plateau value of around 45. Both plateaus suggest a 3-dimensional network of the elastomer, which is usually expected for a covalently cross-linked elastomer only (e.g., the reference elastomer Elastomer-V25). Since covalent crosslinks in the elastomer were not detected by Goff et al[22] and concatenated rings may form through intramolecular reactions[29], topological linkages between concatenated rings are believed to act similar to covalent crosslinks by creating a 3-dimensional network (as shown in Figure 1a).

During the reaction, two separate chains may react intermolecularly to give linear polymers with functional ends (which may further react). In addition, a chain may react intramolecularly to form a cyclic polymer, which is now nonreactive. Concatenation may happen if a linear chain threads one of the cyclic chains followed by ring-closure. In this scenario, isolated rings may form if no chains thread through them. These isolated rings can be extracted by chloroform. On the other hand, a network connected by topological crosslinks, such as a rotaxane-crosslinked elastomer, is known to swell significantly in good solvents due to the inherent chain slipping[30]-[33]. Although the concatenated ring elastomer and the rotaxane-crosslinked elastomer are not exactly identical in terms of their molecular structures, they both possess movable linkages, which may allow the superior swelling ratio of 45 of the concatenated ring elastomer investigated herein. Swelling degrees of conventional silicone networks, even slightly crosslinked networks, do not approach such high values[34]. 


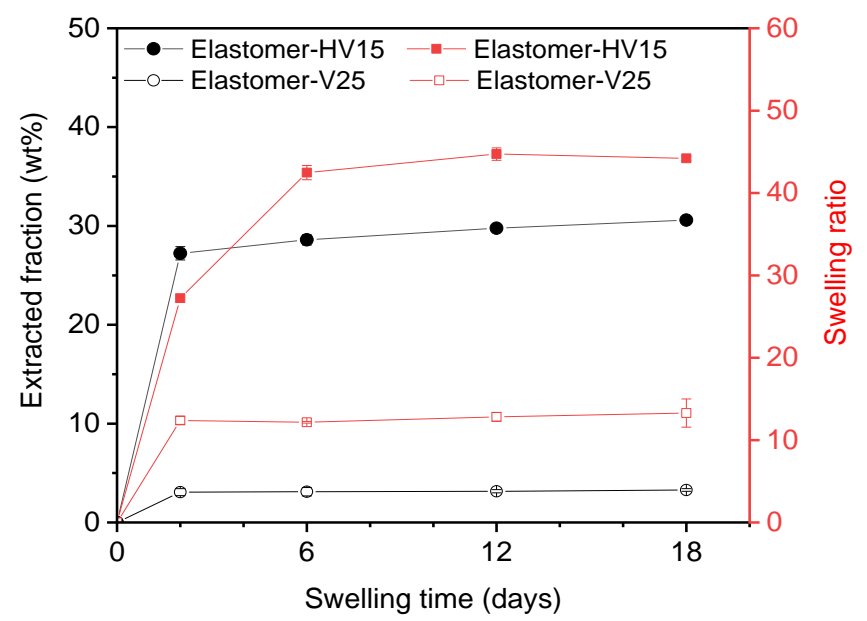

Figure 3. Extracted fraction and swelling ratio of the elastomer (Elastomer-HV15) and the conventional elastomer (Elastomer-V25)

versus swelling time.

\subsection{Linear viscoelasticity}

The linear viscoelasticity of the elastomer was determined at a wide frequency range of $2 \cdot 10^{-4}$ to $10^{2} \mathrm{~Hz}$ (see Figure 4). Overall, the elastomer behaves solid-like since the storage modulus remains larger than the loss modulus for the full frequency range. A relaxation process is observed as the frequency decreases evidenced by the decrease of the storage modulus and the loss modulus. The relaxation is contributed to entangled chain structures, which can release stresses as they slide under a lower shearing frequency. Generally, a polymer network cured from a precursor linear polymer with a chain length $(4000 \mathrm{Da})$ below the entanglement molecular weight is not expected to show a relaxation behavior under the examined frequencies. As the frequency approaches zero, the storage modulus reaches a plateau of $7 \mathrm{kPa}$. The plateau indicates restrictions in the network. Ultra-long linear PDMS melts may also show a pseudo-plateau under the same oscillation conditions, but the plateau value should be identical to the plateau of entangled PDMS (around $200 \mathrm{kPa}[35]$, [36]). Concatenated rings are a possible explanation for the above observations. On one hand, the topological linkages on the concatenated rings possess the same sliding ability as entanglements. That is why the chains in the network can relax as the frequency decreases. One the other hand, the topological linkages cannot be released, which is in contrast to the entanglements in linear chains[37]. Instead, the concatenated rings behave like permanent restrictions, contributing to the constant storage modulus at low frequencies.

Furthermore, the value of $7 \mathrm{kPa}$ of the storage modulus represents an unprecedented low elasticity, only $3.5 \%$ of that for entangled PDMS melts $\left(G_{0}=200 \mathrm{kPa}\right)$. Theoretically, the sol fraction $(\varphi \approx 30 \mathrm{vol} . \%)$ will reduce the shear modulus of the network $(G)$ by $49 \%$ according to the relation of $G=G_{0}(1-\varphi)^{2}[21]$. Thus, the sol fraction is by no means the exclusive contributor to the low shear modulus. A junction in the network is regarded elastically active when more than two of its sites are independently linked to the network. For a conventional cross-linked elastomer, a sufficient number of elastically active junctions can be ensured by a high functionality of a cross-linker and an optimized ratio of reactive groups[6]. However, for the concatenated ring elastomer, rings act as elastically active junctions if they are concatenated with more than two other rings in the network. Dangling ring will be formed if only one other ring in the network concatenate with it. These dangling rings can reduce the shear modulus by acting as internal solvents. 


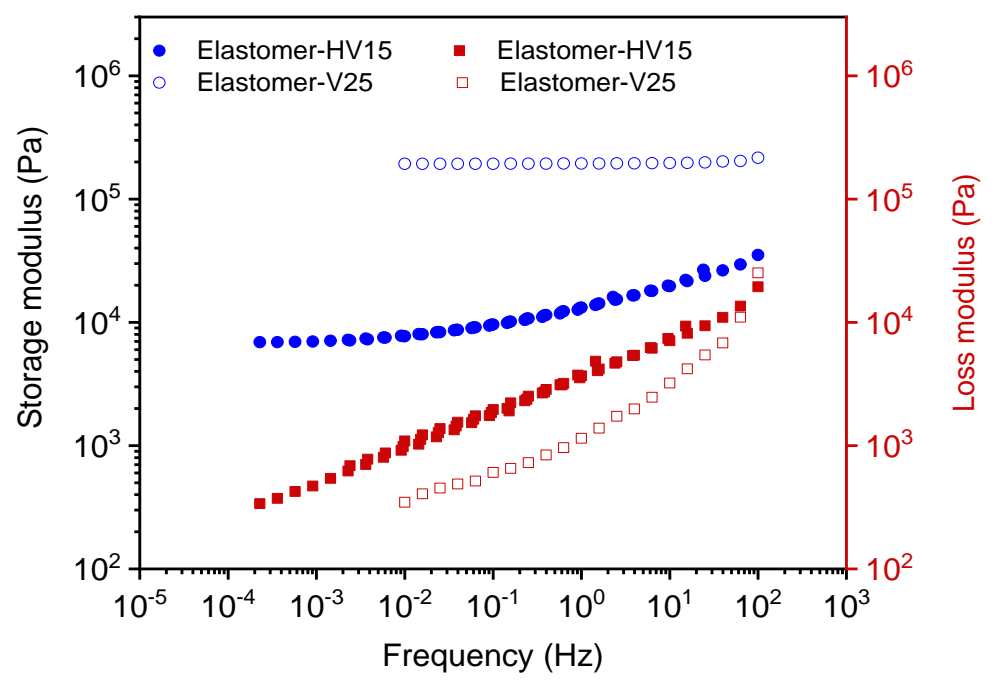

Figure 4. Frequency dependence of storage modulus and loss modulus of the elastomer (Elastomer-HV15) and the conventional elastomer (Elastomer-V25) at room temperature and with an applied strain of $2 \%$.

\subsection{Modeling of stress-strain curve}

Polymer network models are based on the microscopic picture of polymer chains, in more or less details. For example, the affine model[25], one of the classical network models, assumes that polymer chains are only connected by crosslinks, and that all crosslinks move affinely with the deformation. The reduced nominal stress $\left(\sigma_{\text {red }}\right)$ is defined by equation 1 and equals the shear modulus $(G)$.

$$
\begin{gathered}
\sigma_{\text {red }}=\frac{\sigma}{\lambda-\lambda^{-2}} \\
\lambda=\frac{L}{L} \\
\sigma_{\text {red }}=G
\end{gathered}
$$

Where $\sigma$ is the stress and $\lambda$ is the extension ratio. $L$ is the initial length of the specimen and $L^{\prime}$ is the length of the specimen in the stretched state. $G$ is the shear modulus and is determined by linear fitting of $\sigma$ with $\lambda$ in the range of $\lambda=$ 1.0 to $\lambda=1.1$.

Another model, the slip-link model[26], [38] assumes polymer chains are connected by crosslinks and entangled by links which make a sliding contact between polymer networks. The model is expressed by equations (4) and (5).

$$
\begin{gathered}
\sigma_{\text {red }}=G_{c}+G_{s} H(\lambda, \eta) \\
H(\lambda, \eta)=\frac{\lambda^{2}}{\lambda^{2}+\lambda+1}\left[\frac{1}{(\lambda+\eta)^{2}}+\frac{\lambda+1}{\lambda\left(1+\eta \lambda^{2}\right)^{2}}\right]
\end{gathered}
$$

Where $\eta$ is a measure of the freedom of a link to slide compared to the freedom of the movement of a connecting chain ( $\eta$ is commonly set as 0.2343 , a theoretically value based on the assumption that each slip link can on average slide as far as the centres of its topologically neighbouring links[26]). $H(\lambda, \eta)$ is a strain dependent term defined by equation (5). $G_{\mathrm{c}}$ and $G_{\mathrm{s}}$ represent the elastic contributions from cross-links and entanglements, respectively. $G_{\mathrm{c}}$ and $G_{\mathrm{s}}$ are determined by linear fitting of $\sigma_{\text {red }}$ with $H(\lambda, \eta)$ in the range of $H(\lambda, \eta)=0.1$ to $\mathrm{H}(\lambda, \eta)=0.5$. 
In Figure 5 it can be seen that the affine model fits well with the experimental data at strains up to $50 \%$, whereas it overestimates the stress at larger strain. In a concatenated ring network, the topological links on the rings can slide like entanglements to release stress, so-called pseudo-entanglement behavior. When they reach their sliding limit, the topological links behave like crosslinks (so-called pseudo-crosslinks). Therefore, the discrepancy between the affine model and the experimental results can be explained by the pseudo-entanglement behavior of concatenated rings in the network. Generally, the slip-link model is suitable for loosely cross-linked networks where the effect of entanglements are required taken into account. Since concatenated rings possess the properties of both pseudo-crosslinks and pseudoentanglements, the slip-link model was applied for the elastomer as well. As it is expected, the fitting curve based on the slip-link model agrees much better with the experimental data. The fitting parameters of $G_{\mathrm{c}}$ and $G_{\mathrm{s}}$ reflect the contributions from pseudo-crosslinks and pseudo-entanglements, respectively. The results indicate that pseudoentanglements make approximately twice the contribution to the stress as pseudo-crosslinks, since $G_{\mathrm{s}} / G_{\mathrm{c}}=2.1$. Pseudoentanglements contribute to stretch-ability but compromise recovery ability from a stretched state. A higher ratio of $G_{\mathrm{s}}$ $/ G_{\mathrm{c}}$ is expected by increasing the size of rings with larger sliding range of the concatenated rings.

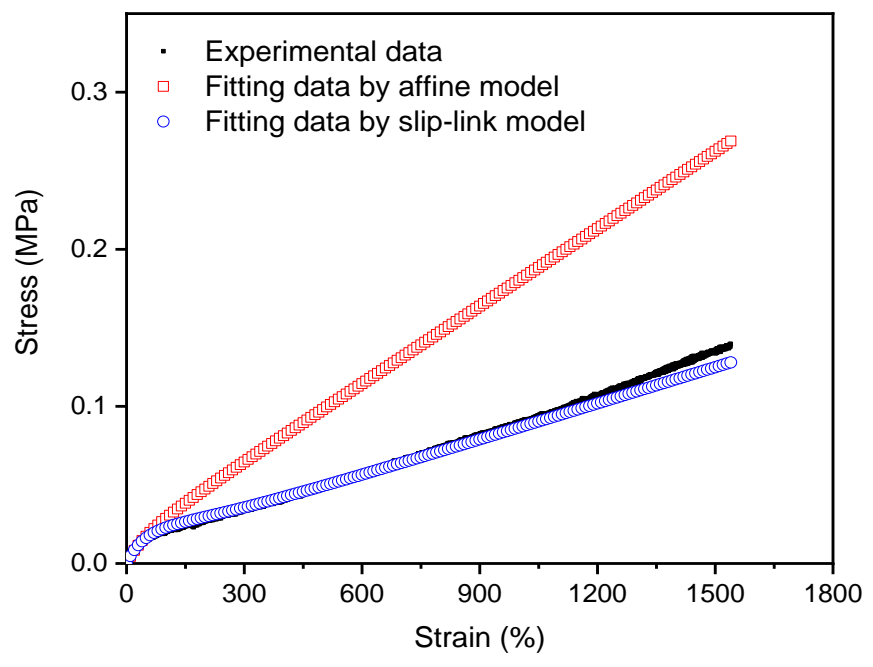

Figure 5. Stress-strain curve of the elastomer (Elastomer-HV15) and the fitting curves respectively based on affine model (fitting shear modulus of $G=16.4 \mathrm{kPa}$ ) and slip-link model (fitting contribution from pseudo-entanglements of $G_{\mathrm{s}}=15.5 \mathrm{kPa}$, and fitting contribution from pseudo-crosslinks of $G_{\mathrm{c}}=7.4 \mathrm{kPa}$ ).

\section{CONCLUSION}

The unprecedented soft silicone elastomer is hypothesized to be created by concatenated rings. A series of characterizations support the hypothesis and provide details in molecular structures.

Long-term swelling experiment shows that the gel fraction (70 wt\%) of the elastomer remains constant even after being swollen in a good solvent for 18 days, indicating that the network is truly linked in a covalent manner. This can be explained by concatenated rings, which act as cross-links responsible for network formations.

A similar frequency dependence of storage modulus and loss modulus of the elastomer as that of conventionally crosslinked elastomers is shown by linear viscoelastic (LVE) analysis. The distinct feature is the unprecedented low plateau of storage modulus $(7 \mathrm{kPa})$ at low frequencies, accounting for only $3.5 \%$ entanglement threshold $(200 \mathrm{kPa})$. Isolated rings and dangling rings contribute to the low plateau modulus, respectively acting as external solvents and internal solvents.

Both swelling experiment and LVE analysis indicate that the concatenated rings possess the dynamic properties of pseudo-entanglements and pseudo-crosslinks. On one hand, concatenated rings demonstrate the sliding ability, similar to that of entanglements, which endows the rings a partly relaxation ability. On the other hand, the concatenated rings 
exhibits a permanent linkage behavior, like crosslinks, due to their sliding limits. The modeling of the stress-strain behavior based on the slip-link model indicates that the contribution to elasticity from pseudo-entanglements is twice that from pseudo-crosslinks in the given system.

Generating a higher fraction of dangling rings, is expected to create even softer dielectric elastomers. Therefore, additional control over the ring formation is expected to open up for a wide range of unprecedented properties of silicone elastomers to be used for dielectric elastomers, amongst others. Furthermore, due to the used chemistry, the silicone elastomers can be combined with electrodes identical to that of common silicones, therefore not opening up for requirements of novel types of electrodes[39].

\section{REFERENCES}

[1] Romasanta, L. J., Lopez-Manchado, M. A. and Verdejo, R., "Increasing the performance of dielectric elastomer actuators: A review from the materials perspective," Prog. Polym. Sci. 51, 188-211 (2015).

[2] Brochu, P. and Pei, Q., "Advances in dielectric elastomers for actuators and artificial muscles," Macromol. Rapid Commun. 31(1), 10-36 (2010).

[3] Yang, Y., Wang, K.,Gu, X. and Leong, K. W., "Biophysical regulation of cell behavior — cross talk between substrate stiffness and nanotopography," Engineering 3(1), 36-54 (2017).

[4] Madsen, F. B., Daugaard, A. E., Hvilsted, S. and Skov, A. L., "The current state of silicone-based dielectric elastomer transducers," Macromol. Rapid Commun. 37(5), 378-413 (2016).

[5] Bar-Cohen, Y., "Humanlike robots: state of the art and challenges," Proc. SPIE 10965, 1096507 (2019).

[6] Mazurek, P., Vudayagiri, S. and Skov, A. L., "How to tailor flexible silicone elastomers with mechanical integrity: A tutorial review," Chem. Soc. Rev. 48(6), 1448-1464 (2019).

[7] Skov, A. L. and Yu, L., "Optimization techniques for improving the performance of silicone-based dielectric elastomers," Adv. Eng. Mater. 20(5), 1700762 (2018).

[8] Mazich, K. A. and Samus, A., "Role of entanglement couplings in threshold fracture of a rubber network," Macromolecules 23(9), 2478-2483 (1990).

[9] Madsen, F. B., Zakaria, S., Yu, L. and Skov, A. L., "Mechanical and electrical ageing effects on the long-term stretching of silicone dielectric elastomers with soft fillers," Adv. Eng. Mater.18(7), 1154-1165 (2016).

[10] Lenhart, J. L. and Cole, P. J., "Adhesion properties of lightly crosslinked solvent-swollen polymer gels," J. Adhes. 82(10), 945-971 (2006).

[11] Larsen, A. L., Sommer-Larsen, P. and Hassager, O., "How to tune rubber elasticity," Proc. SPIE 5385, 108 (2004).

[12] Hunt, S., McKay, T. G. and Anderson, I. A., “A self-healing dielectric elastomer actuator,” Appl. Phys. Lett. 104(11), 3-6 (2014).

[13] Zakaria, S., Yu, L., Kofod, G. and Skov, A. L., "The influence of static pre-stretching on the mechanical ageing of filled silicone rubbers for dielectric elastomer applications," Mater. Today Commun. 4, 204-213 (2015).

[14] Heinrichs, V., Dieluweit, S., Stellbrink, J., Pyckhout-Hintzen, W., Hersch, N., Richter, D. and Merkel, R., "Chemically defined, ultrasoft PDMS elastomers with selectable elasticity for mechanobiology," PLOS One 13(4), 1-22 (2018).

[15] Yang, S., Kim, D. Bahk, Kataruka, J., A., Dunn, A. C. and Hutchens, S. B., "Hydraulic fracture geometry in ultrasoft polymer networks,” Int. J. Fract. 219(1), 89-99 (2019).

[16] Glover, J. D., McLaughlin, C. E., McFarland, M. K. and Pham, J. T., "Extracting uncrosslinked material from low modulus sylgard 184 and the effect on mechanical properties," J. Polym. Sci. 58(2), 343-351 (2020).

[17] Yu, L., Madsen, F. B., Hvilsted, S. and Skov, A. L., "Dielectric elastomers, with very high dielectric permittivity, based on silicone and ionic interpenetrating networks," RSC Adv. 5(61), 49739-49747 (2015).

[18] Liang, H., Morgan, B. J., Xie, G., Martinez, M. R., Zhulina, E. B., Matyjaszewski, K., Sheiko, S. S. and Dobrynin, A. V., "Universality of the entanglement plateau modulus of comb and bottlebrush polymer melts," Macromolecules 51(23), 10028-10039 (2018).

[19] Cai, L. H., Kodger, T. E., Guerra, R. E., Pegoraro, A. F., Rubinstein M. and Weitz, D. A., "Soft poly(dimethylsiloxane) elastomers from architecture-driven entanglement free design," Adv. Mater. 27(35), 5132-5140 (2015).

[20] Vasiliev, V. G., Rogovina, L. Z. and Slonimsky, G. L., "Dependence of properties of swollen and dry polymer 
networks on the conditions of their formation in solution," Polymer 26(11), 1667-1676 (1985).

[21] Urayama, K., Kawamura,T. and Kohjiya, S., "Structure-mechanical property correlations of model siloxane elastomers with controlled network topology," Polymer 50(2), 347-356 (2009).

[22] Goff, J., Sulaiman, S., Arkles, B. and Lewicki, J. P., "Soft materials with recoverable shape factors from extreme distortion states," Adv. Mater. 28(12), 2393-2398 (2016).

[23] Sachot, N., Engel, E. and Castano, O., "Hybrid organic-inorganic scaffolding biomaterials for regenerative therapies," Curr. Org. Chem. 18(18), 2299-2314 (2014).

[24] Niu, Z. and Gibson, H. W., "Polycatenanes," Chem. Rev. 109 (11), 6024-6046 (2009).

[25] Rubinstein, M. and Panyukov, S., "Elasticity of polymer networks," Macromolecules 35(17), 6670-6686 (2002).

[26] Road, M., "Elasticity of entangled networks," Polymer 22(4), 1010-1018 (1981).

[27] Vaicekauskaite, J., Mazurek, P., Vudayagiri, S. and Skov, A. L., "Mapping the mechanical and electrical properties of commercial silicone elastomer formulations for stretchable transducers," J. Mater. Chem. C 8(4), 1273-1279 (2020).

[28] Park, S., Mondal, K., Treadway, T. M., Kumar. V., Ma, S., Holbery, J. D. and Dickey, M. D., "Silicones for stretchable and durable soft devices: beyond sylgard-184," ACS Appl. Mater. Interfaces 10(13), 11261-11268 (2018).

[29] Stefano, S. Di and Ercolani, G., "Statistical ring catenation under thermodynamic control: Should the JacobsonStockmayer cyclization theory take into account catenane formation?," J. Phys. Chem. B, 121(3), 649-656 (2017).

[30] Koyama, Y., "Synthesis of topologically crosslinked polymers with rotaxane-crosslinking points," Polymer Journal 46(6), 315-322 (2014).

[31] Liu, C., Kadono, H., Mayumi, K., Kato, K., Yokoyama, H. and Ito, K., "Unusual fracture behavior of slide-ring gels with movable cross-links," ACS Macro Lett. 6(12), 1409-1413 (2017).

[32] Ishida, K., Uno, T., Itoh, T. and Kubo, M., "Synthesis and property of temperature-responsive hydrogel with movable cross-linking points," Macromolecules 45(15), 6136-6142 (2012).

[33] Miki, K., Inamoto, Y., Inoue, S., Uno, T., Itoh, T. and Kubo, M., "Preparation of three-dimensional poly(dimethylsiloxane) (PDMS) with movable cross-linking,” J. Polym. Sci. Part A Polym. Chem. 47(21), 58825890 (2009).

[34] Frankær, S. M. G., Jensen, M. K., Bejenariu, A. G. and Skov, A. L., "Investigation of the properties of fully reacted unstoichiometric polydimethylsiloxane networks and their extracted network fractions," Rheol. Acta 51(6), 559-567 (2012).

[35] Gottlieb, M., Macosko, C. W., Benjamin, G. S., Meyers, K. O. and Merrill, E. W., "Equilibrium modulus of model poly(dimethylsiloxane) networks" Macromolecules 14(4), 1039-1046 (1981).

[36] Lomellini, P., "Effect of chain length on the network modulus and entanglement," Polymer 33(6), 1255-1260 (1992).

[37] Neuefeind, J., Skov, A. L., Daniels, J. E., Honkimaki, V., Jakobsen, B., Oddershede, J. and Poulsen, H. F., “A multiple length scale description of the mechanism of elastomer stretching," RSC Adv. 6(98), 95910-95919 (2016).

[38] Weil, T. and Thirion, P., "Assessment of the sliding link model of chain entanglement in polymer networks," Polymer 25(5), 609-614 (1984).

[39] Rosset, S. and Shea, H. R., "Flexible and stretchable electrodes for dielectric elastomer actuators," Appl. Phys. A Mater. Sci. Process.110(2), 281-307 (2013). 